\title{
Synthesis of Graphene-CdSe Composite by a Simple Hydrothermal Method and Its Photocatalytic Degradation of Organic Dyes
}

\author{
OH Won-Chun ${ }^{1, *}$, CHEN Mingliang ${ }^{1}$, CHO Kwangyoun ${ }^{2}$, KIM Cheolkyu $^{3}$, MENG Zeda $^{1}$, ZHU Lei $^{1}$ \\ ${ }^{1}$ Department of Advanced Materials Science \& Engineering, Hanseo University, Chungnam 356-706, Korea \\ ${ }^{2}$ Korea Institute of Ceramic Engineering and Technology, Seoul 153-801, Korea \\ ${ }^{3}$ Department of Environmental Engineering, Hanbat National University, Dajeon 305-719, Korea
}

\begin{abstract}
A graphene-CdSe composite was synthesized by a facile hydrothermal method, and characterized by X-ray diffraction, scanning electron microscopy with energy dispersive X-ray analysis, transmission electron microscopy and UV-vis diffuse reflectance spectrophotometry. The graphene-CdSe composite efficiently catalyzed the photodegradation of methylene blue (MB), methyl orange (MO) and rhodamine B (Rh.B) in aqueous solution under UV or visible light irradiation. The graphene-CdSe composite exhibited a higher photocatalytic activity for the MB solution.
\end{abstract}

Key words: graphene; cadmium selenide; organic dye; photocatalytic activity

CLC number: $0643 \quad$ Document code: A

Received 7 June 2011. Accepted 10 July 2011.

*Corresponding author. Tel: +82-41-660-1337; Fax:+82-41-688-3352; E-mail: wc_oh@hanseo.ac.kr

English edition available online at Elsevier ScienceDirect (http://www.sciencedirect.com/science/journal/18722067).

Recently, it was found that carbon nanostructures (such as $\mathrm{C}_{60}$ and carbon nanotubes), which are excellent electron acceptors and highly conductive scaffolds, are useful in photovoltaic conversion and photocatalysis [1-5]. Graphene, a flat monolayer of hexagonally arrayed $s p^{2}$-bonded carbon atoms tightly packed into a two-dimensional (2D) honeycomb lattice, is a major focus of recent research due to its outstanding mechanical, electrical, thermal, and optical properties [6-9]. It has potential applications in many different areas [10-14]. The useful properties of graphene can be extended by integrating it with other nanomaterials to form unique hybrid materials. The fabrication of graphene materials decorated by metal nanoparticles is expected to give graphene nanocrystal hybrids with enhanced properties such as high catalytic activity and excellent electrochemical properties. Recently, semiconductor nanoparticles such as $\mathrm{TiO}_{2}, \mathrm{ZnO}$, and $\mathrm{CdS}$ have also been utilized to decorate graphene nanosheets to prepare graphene-based nanomaterials with remarkable optical and photovoltaic properties [15-17].

Cadmium selenide (CdSe) is an n-type semiconductor. Its band gap energy was reported to be from 1.65 to $1.8 \mathrm{eV}$ [18-21]. It was found to be suitable for various optoelectronic applications in catalysis [22], solar cells [23], and biological labeling [24]. More recently, many groups have synthesized CdSe nanomaterials with high photocatalytic activity for the degradation of organic pollutants under UV light irradiation, such as CdSe-Pt nanorods and nanonets
[24], hybrid CdSe-Au nanodumbbells [25], and CdSe/ZnSphotosensitized nano- $\mathrm{TiO}_{2}$ films [26]. However, few papers have discussed the preparation and properties of CdSe combined with graphene composites and their application in the degradation of organic dyes.

In this paper, we report the synthesis of graphene nanosheets coated with CdSe nanoparticles obtained directly by a hydrothermal preparation method. The composite was characterized by X-ray diffraction (XRD), scanning electron microscopy (SEM) with energy dispersive X-ray (EDX) analysis, transmission electron microscopy (TEM), and UV-Vis diffuse reflectance spectroscopy. The photocatalytic activity of the graphene-CdSe composite was evaluated by the degradation of different organic dyes, methylene blue (MB), methyl orange (MO), and rhodamine $\mathrm{B}$ (Rh.B), in aqueous solution under irradiation of UV or visible light.

\section{Experimental}

\subsection{Synthesis of the graphene-CdSe composite}

First, $\mathrm{Na}_{2} \mathrm{SO}_{3}$ (5 g, Duksan Pharmaceutical Co., Ltd, Korea) and selenium metal powder ( $0.5 \mathrm{~g}$, DaeJung Chemicals \& Metal Co., Ltd, Korea) were dissolved in $30 \mathrm{ml}$ distilled water and refluxed for $1 \mathrm{~h}$ to form $\mathrm{Na}_{2} \mathrm{SeSO}_{3}$ solution. $\mathrm{Cd}\left(\mathrm{CH}_{3} \mathrm{COO}\right)_{2}(0.5 \mathrm{~g}$, DaeJung Chemicals \& Metal Co., Ltd, Korea) was dissolved in $2 \mathrm{ml}$ distilled water. $\mathrm{NH}_{4} \mathrm{OH}(6 \mathrm{ml}$, 
28\%, DaeJung Chemicals \& Metal Co., Ltd, Korea) was added to it and the mixture was stirred till it dissolved completely to form a $\mathrm{Cd}\left(\mathrm{NH}_{3}\right)_{4}{ }^{2+}$ solution. Graphene oxide $(0.5$ g) prepared by the Hummers-Offeman method in our previous work [27] was added into the $\mathrm{Na}_{2} \mathrm{SeSO}_{3}$ solution and $\mathrm{Cd}\left(\mathrm{NH}_{3}\right)_{4}{ }^{2+}$ solution under stirring to allow the formation of hydrogen bonds between the CdSe nanocrystals and graphene oxide. After hydrothermal reaction at $80{ }^{\circ} \mathrm{C}$ for $5 \mathrm{~h}$, graphene oxide was reduced to graphene nanosheets and
CdSe compounds were grown on its surface to generate a graphene-CdSe composite. The preparation procedure is shown in Fig. 1. Finally, the temperature of the mixture was brought down to room temperature and the mixture was filtered through Whatman filter paper. The solid obtained was collected and washed with distilled water five times. After drying under vacuum at $100{ }^{\circ} \mathrm{C}$ for $8 \mathrm{~h}$, the graphene-CdSe composite was obtained.

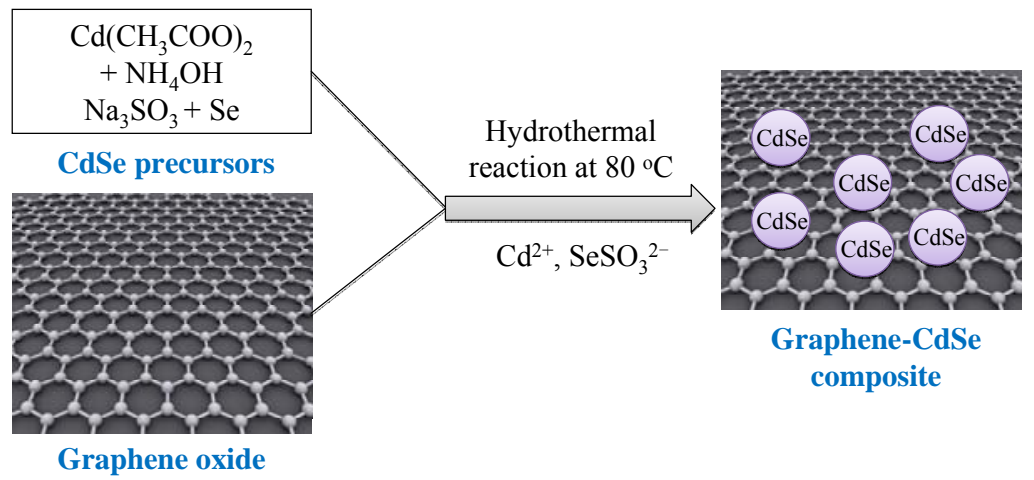

Fig. 1. Synthesis of graphene-CdSe composite by a simple hydrothermal method.

\subsection{Characterization of the graphene-CdSe composite}

XRD (Shimadzu XD-D1) using monochromatic high intensity $\mathrm{Cu} K_{\alpha}$ radiation $(\lambda=0.15406 \mathrm{~nm})$ was used to characterize the crystallinity. SEM (JSM-5600) was used to observe the surface state and structure. TEM (Jeol, JEM- 2010, Japan) was used to determine the state and particle size of the composite. TEM at an acceleration voltage of $200 \mathrm{kV}$ was used to investigate the number and stacking state of the graphene layers of the various samples. TEM specimens were prepared by placing a few drops of sample solution on a carbon grid. The element mapping of regions of the composite was performed by EDX analysis (attached to the SEM). UV-Vis diffuse reflectance spectra were obtained using a UV-Vis spectrophotometer (Neosys-2000) by using $\mathrm{BaSO}_{4}$ as a reference at room temperature. These were converted from reflection to absorbance by the KubelkaMunk method.

\subsection{Photocatalytic studies}

The photocatalytic tests were performed at room temperature. MB (99.99+\%), MO (99.99+\%), and Rh.B $(99.99+\%)$ used as model pollutants were purchased from Duksan Pure Chemical Co., Ltd, Korea, Daejung Chemicals \& Metals Co., Ltd, Korea, and Samchun Pure Chemical Co., Ltd, Korea, respectively. Table 1 shows the structure and properties of MB, MO, and Rh.B. Graphene-CdSe composite $(0.03 \mathrm{~g})$ was added into $50 \mathrm{ml}$ of $10 \mu \mathrm{mol} / \mathrm{L}$ (designated as $c_{0}$ ) organic dye (MB, MO, or Rh.B) solution. This was maintained in the dark for $2 \mathrm{~h}$ to ensure the adsorption/desorption equilibrium of the organic dye. Then, the solution was irradiated with a UV or visible lamp. The first sample was taken at the end of the dark adsorption period (just before the light was turned on) to determine the MB concentration in the solution. This was treated as the starting point $(t=0)$ of the reaction when the concentration of

Table 1 Molecular structure and absorbance maximum $\left(\lambda_{\max }\right)$ of the organic dyes

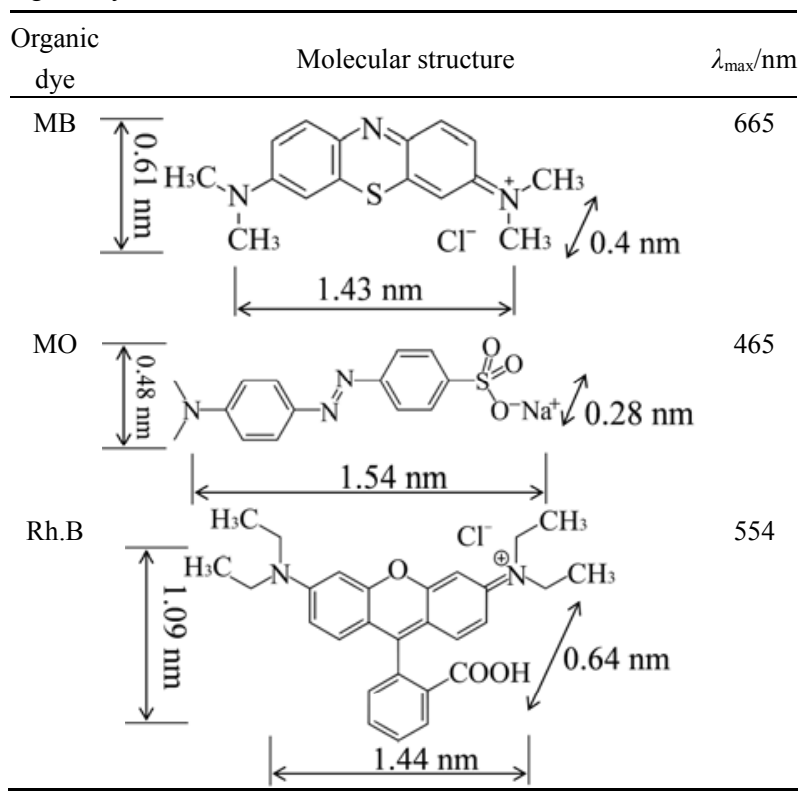


the organic dye was $c_{\text {ads }}$. Samples were withdrawn regularly from the reactor at $30,60,90,120,180$, and $240 \mathrm{~min}$ and immediately centrifuged to separate any suspended solid before analysis. The concentration of organic dye $(c)$ in the solution during the photocatalytic degradation reaction was monitored by measuring the absorbance of the solution samples with a UV-Vis spectrophotometer (Optizen POP) at $\lambda_{\max }=665,465$, and $554 \mathrm{~nm}$, respectively, for $\mathrm{MB}, \mathrm{MO}$, and Rh.B since no reaction products absorb at these wavelength and by using a calibration curve. The degradation efficiency $(\eta \%)$ was calculated as

$$
\eta(\%)=\left(1-c / c_{0}\right) \times 100
$$

where $c$ is the concentration of organic dye at time $t$.

\section{Results and discussion}

\subsection{Characterization results}

Figure 2 shows the XRD patterns of graphene, CdSe, and the graphene-CdSe composite. The diffractogram of graphene exhibited the typical peaks at $2 \theta=25.9^{\circ}$ and $42.7^{\circ}$ corresponding to the graphite (002) and (100) reflections (JCPDS 01-0646) [28], respectively. For the CdSe compound, the XRD diffraction peaks at $2 \theta$ of $25.4^{\circ}, 42^{\circ}$, and $49.6^{\circ}$ can be indexed to the characteristic peaks of the (111), (220), and (311) plane reflections of cubic crystal structure CdSe with lattice constants of $0.605 \mathrm{~nm}$ according to the standard powder diffraction data (JCPDS 65-2891 for CdSe, cubic) $[29,30]$. However, for the graphene-CdSe composite, only the peaks from CdSe were detected. The (002) and (100) reflection of graphene overlapped the cubic (111) and (200) reflection of CdSe. Therefore, the intensity of the peaks at $2 \theta=25.4^{\circ}$ and $42^{\circ}$ from the graphene-CdSe composite was stronger than that from the CdSe compound, and

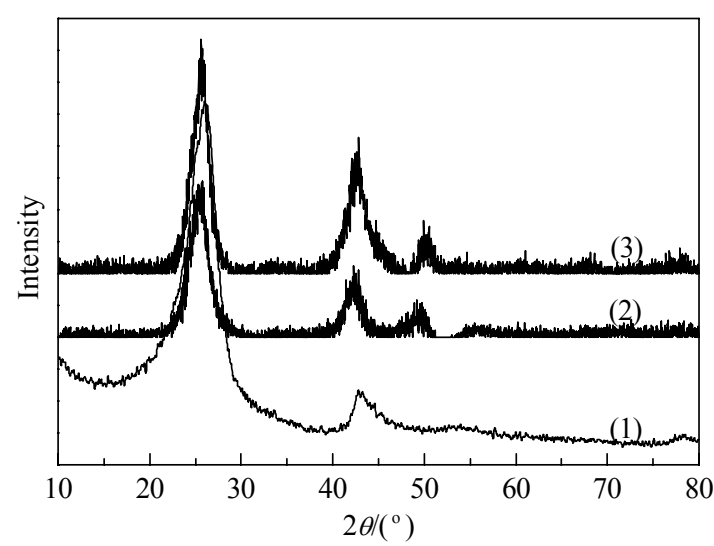

Fig. 2. XRD patterns of graphene (1), CdSe compound (2), and graphene-CdSe composite (3).

the micromorphology of graphene-CdSe was different from that of the mixture of graphene and CdSe. No peak for graphene oxide or impurities was detected, indicating that the hydrothermal method used in this study gave the formation of a graphene-CdSe composite and the graphene oxide was reduced completely after reaction with CdSe. This result agrees with previous works [31,32] which used graphene oxide as the support material to prepare graphene based nanomaterials.

Figure 3(a) and (b) shows the SEM microphotographs of the graphene-CdSe composite. Very uniform spherical-shaped CdSe particles and agglomerates were observed. Spherical-shaped agglomerated CdSe particles were coated on the surface of the graphene. More detailed information of the surface state and particle size were obtained by TEM (Fig. 3(c)). The 2D structure of the graphene sheet and the surface was very smooth. CdSe particles and agglomerates were coated on the surface of the graphene sheet.
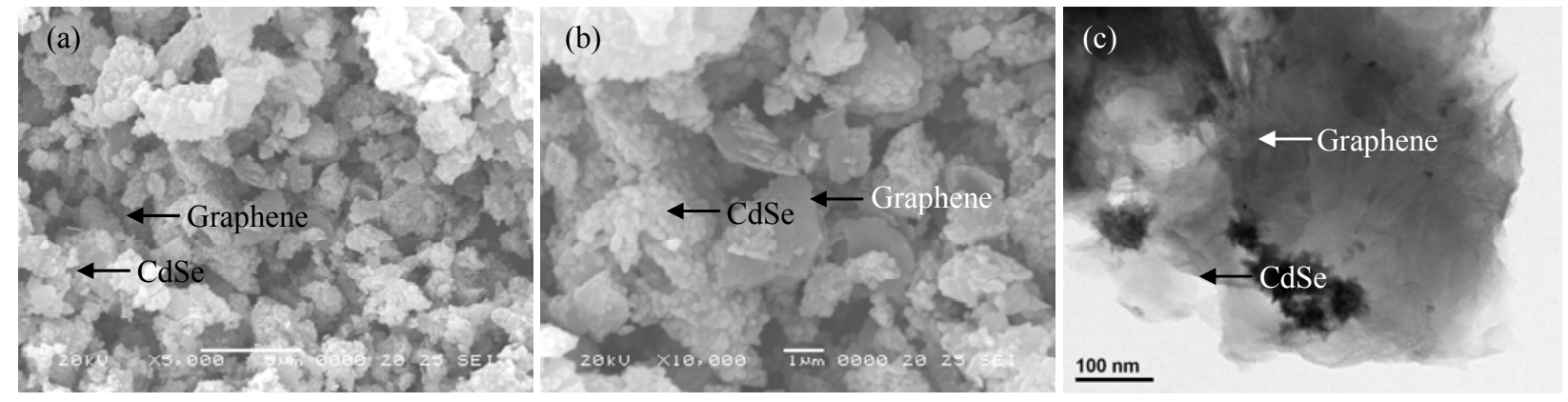

Fig. 3. SEM (a and b) and TEM (c) images of the graphene-CdSe composite.

To get information on the elements and element mass, the graphene-CdSe composite was examined by EDX. Figure 4 shows the EDX microanalysis and element mass of the graphene-CdSe composite. The main elements such as $\mathrm{C}, \mathrm{Cd}$, and Se were present. The strong $\mathrm{C}$ signal mainly originated from the graphene nanosheets. The $\mathrm{Cd}$ and Se peak came from the precursor materials $\mathrm{Cd}\left(\mathrm{CH}_{3} \mathrm{COO}\right)_{2}$ and selenium metal powder.

Figure 5 shows the UV-Vis diffuse reflectance spectra of $\mathrm{CdSe}$ and the graphene-CdSe composite. The reflectance characteristics of the CdSe compound were quite similar to that of the graphene-CdSe composite except that the CdSe 

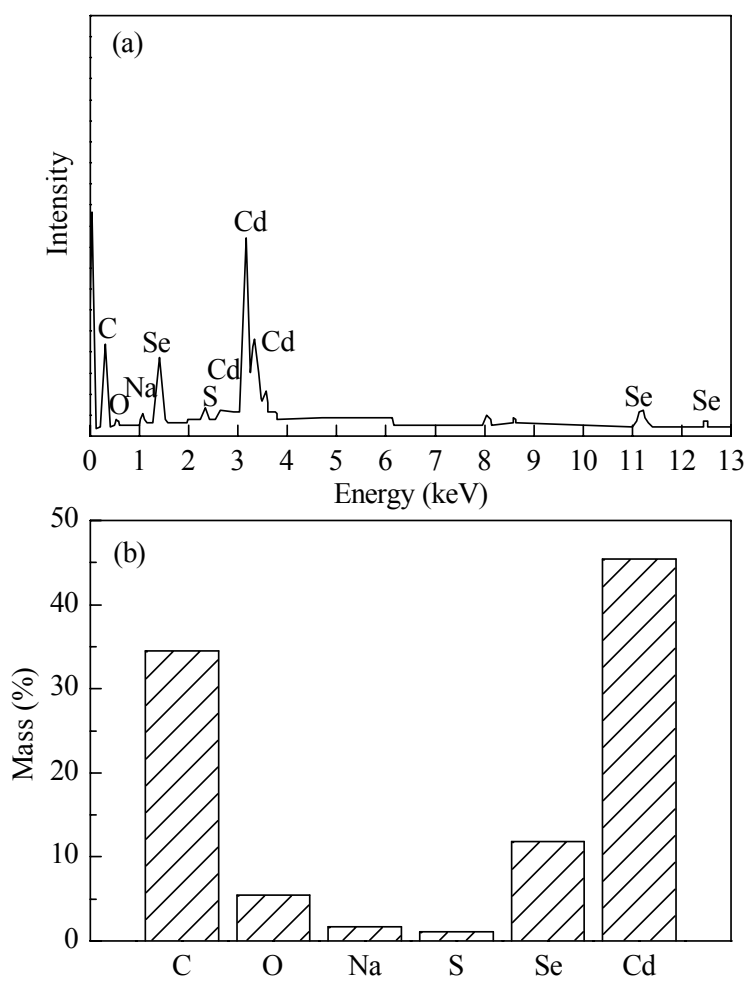

Fig. 4. EDX microanalysis (a) and element mass (b) of the graphene-CdSe composite.

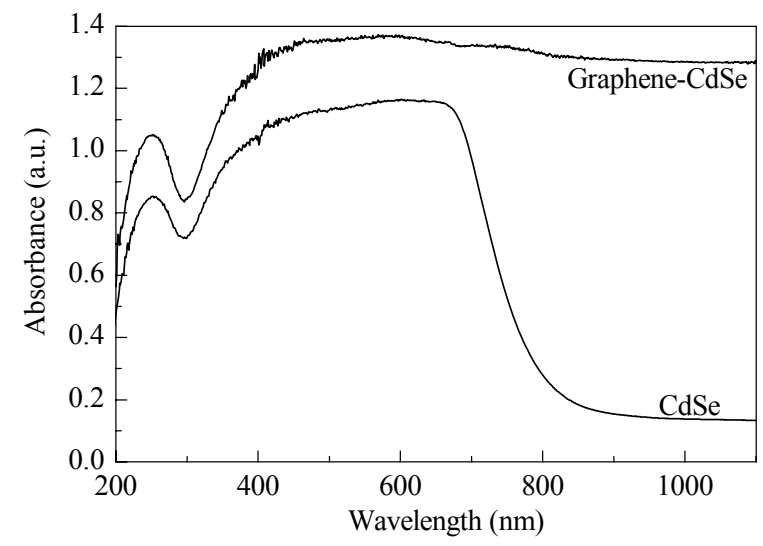

Fig. 5. UV-Vis diffuse reflectance spectra of CdSe and the graphene-CdSe composite.

compound has an absorption edge at $830 \mathrm{~nm}$. We calculate the band gap energy of CdSe by $\alpha h v=A\left(h v-E_{\mathrm{g}}\right)^{1 / 2}$, where $\alpha$, $v, E_{\mathrm{g}}$, and $A$ are the absorption coefficient, light frequency, band gap, and a constant, respectively. Therefore, the band gap energy $\left(E_{\mathrm{g}}\right)$ of the $\mathrm{CdSe}-\mathrm{TiO}_{2}$ composite can be estimated from a plot of $(\alpha h v)^{1 / 2}$ versus photon energy $(h v)$. The band gap energy of CdSe was $1.74 \mathrm{eV}$, which was close to the literature value of 1.65 to $1.8 \mathrm{eV}(\mathrm{CdSe})$ [18-21].

Moreover, the two composites both exhibited strong absorption in the UV light region at wavelengths less than 400 $\mathrm{nm}$ and the visible light region at wavelengths at 400-800 $\mathrm{nm}$, which were assigned to CdSe. The absorption of the graphene-CdSe composite was higher than that of the CdSe compound in both UV light and visible light regions, indicating the graphene-CdSe composite would exhibit better photoactivity than the CdSe compound.

\subsection{Degradation of organic dyes solution}

The degradation of organic dye can be divided to two parts: adsorption in the dark for $120 \mathrm{~min}$ and photocatalysis under light irradiation for $240 \mathrm{~min}[33,34]$. The effects of reaction variables such as organic dye, initial concentration, catalyst amount, light source, etc. on the degradation efficiency were studied. The results are given below.

\subsubsection{Effect of different organic dyes}

The effect of different organic dyes on degradation efficiency was investigated by using the concentration of 10 $\mu \mathrm{mol} / \mathrm{L}$ under $240 \mathrm{~min}$ UV irradiation with a catalyst amount of $0.03 \mathrm{~g}$. The results are shown in Fig. 6. The graphene-CdSe composite achieved almost $100 \%$ MB removal after adsorption for $120 \mathrm{~min}$ in the dark, while $\mathrm{MO}$ and Rh.B were removed by only $6 \%$ and $19 \%$, respectively. From Table 1, it is clear that the molecule size of MB is much smaller than that of MO and Rh.B, which explained why the graphene-CdSe composite showed the best adsorption effect for the MB solution over the MO and Rh.B solutions. For the photocatalytic effect, the $\mathrm{MO}$ in the solution was decreased $40 \%$ after 240 min while Rh.B in the solution was decreased by only $20 \%$ for the same irradiation time. The result indicates that the graphene-CdSe composite gave the best degradation for the MB solution. So, we chose the MB solution with which to perform further experiments.

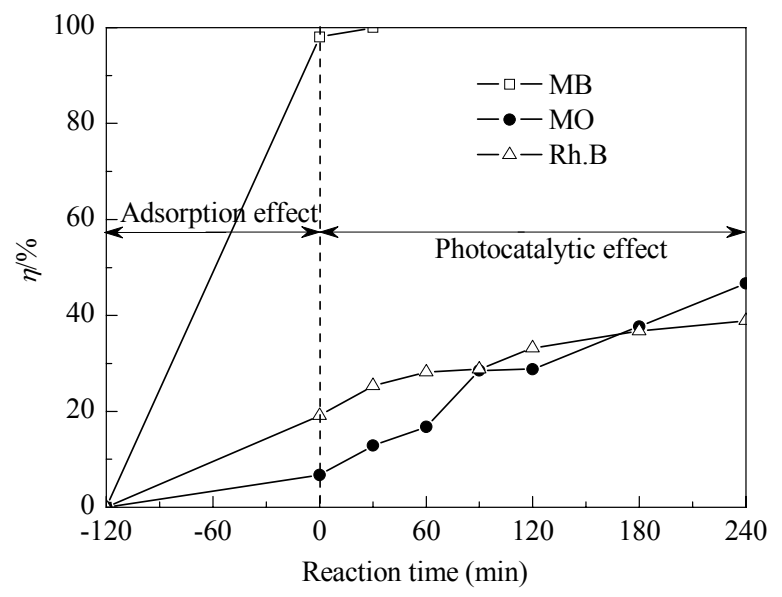

Fig. 6. Degradation efficiency of different organic dyes by the graphene-CdSe composite under UV irradiation. The concentration of organic dye was $10 \mu \mathrm{mol} / \mathrm{L}$. The amount of graphene-CdSe composite was $0.03 \mathrm{~g}$. 


\subsubsection{Effect of catalyst amount}

Experiments were performed to study the rate of degradation with different catalyst amounts ranging from 0.01 to $0.03 \mathrm{~g}$ for a MB solution with a concentration of $10 \mu \mathrm{mol} / \mathrm{L}$. The results are shown in Fig. 7. The degradation efficiency of the MB solution increased sharply with catalyst amount. This was due to an increased number of adsorption and catalytic sites on the surface of the graphene-CdSe composite. The result indicated that the optimum catalyst amount for the degradation of MB solution was $0.03 \mathrm{~g}$.

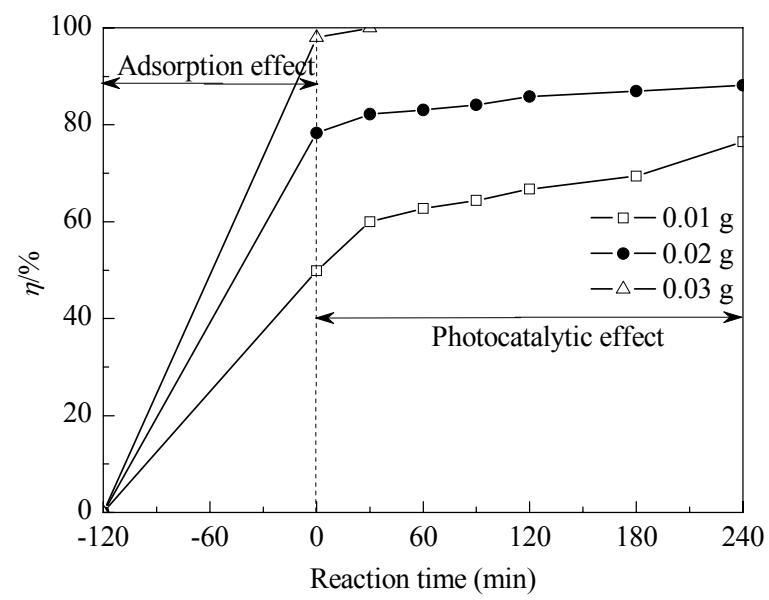

Fig. 7. Degradation efficiency of MB solution by the graphene-CdSe composite under UV irradiation with different amounts of graphene-CdSe composite. The concentration of MB was $10 \mu \mathrm{mol} / \mathrm{L}$.

\subsubsection{Effect of MB concentration}

The effect of MB concentration on degradation efficiency was investigated by varying the concentration from 100 to $10 \mu \mathrm{mol} / \mathrm{L}$ under $240 \mathrm{~min} \mathrm{UV}$ irradiation with a catalyst amount of $0.03 \mathrm{~g}$. The results are shown in Fig. 8. The deg-

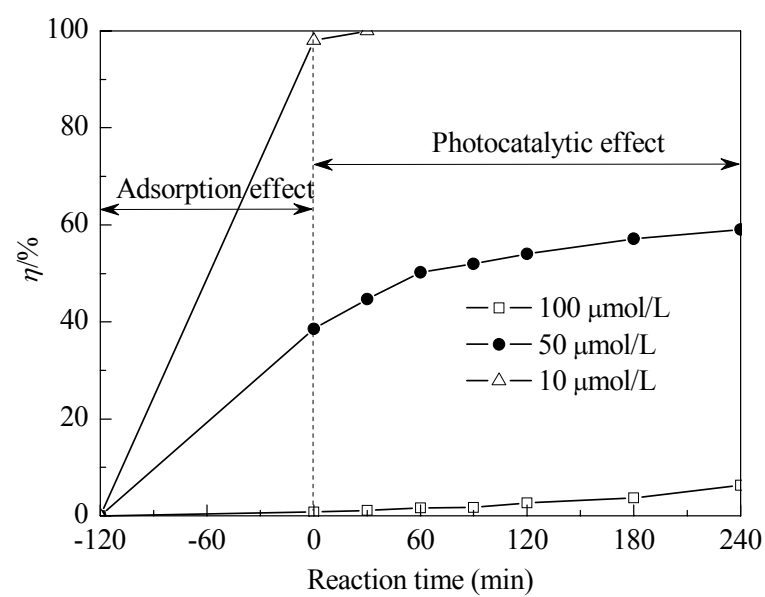

Fig. 8. Degradation efficiency of MB solution with different concentrations by the graphene-CdSe composite under UV irradiation. The amount of graphene-CdSe composite was $0.03 \mathrm{~g}$. radation efficiency decreased with an increase in concentration of MB from 100 to $10 \mu \mathrm{mol} / \mathrm{L}$. After UV irradiation for $240 \mathrm{~min}$, the MB solution with a concentration of $50 \mu \mathrm{mol} / \mathrm{L}$ had $20 \%$ dye removed while the MB solution with a concentration of $100 \mu \mathrm{mol} / \mathrm{L}$ had only $5.6 \%$ dye removed. One possible reason is that at a high $\mathrm{MB}$ concentration, some UV light is absorbed by MB molecules in the aqueous solution rather than by the catalyst particles, which reduced the efficiency for the catalytic reaction. Another possible reason is that the intermediate products formed upon the photocatalytic degradation of $\mathrm{MB}$ compete with $\mathrm{MB}$ molecules for the limited adsorption and catalytic sites on the surface of the catalyst particles, and thus inhibited the degradation of MB to some extent. This result indicated that the optimum $\mathrm{MB}$ concentration was $50 \mu \mathrm{mol} / \mathrm{L}$.

\subsubsection{Effect of light source}

The photocatalytic activity of the graphene-CdSe composite was performed by the degradation of MB solution (50 $\mu \mathrm{mol} / \mathrm{L})$ under $240 \mathrm{~min}$ of $\mathrm{UV}$ light $(6 \mathrm{~W}, \lambda=365 \mathrm{~nm})$ and visible light $(8 \mathrm{~W}, \lambda \geq 420 \mathrm{~nm})$ irradiation with a catalyst amount of $0.03 \mathrm{~g}$. The results are shown in Fig. 9. The results clearly showed that the MB degradation efficiency under UV irradiation for $240 \mathrm{~min}$ was $18 \%$ while under visible light irradiation for the same time it was $35 \%$. This is due to that the power of the visible light $(8 \mathrm{~W})$ was higher than that of the UV light (6W). It also indicated that the graphene-CdSe composite showed photocatalytic activity not only under UV light but also under visible light. Photocatalytic reactions on the graphene-CdSe composite surface can be expressed by the Langmuir-Hinshelwood model [35]. The photocatalytic degradation of $\mathrm{MB}$ by the graphene-CdSe composite under UV and visible light obeyed

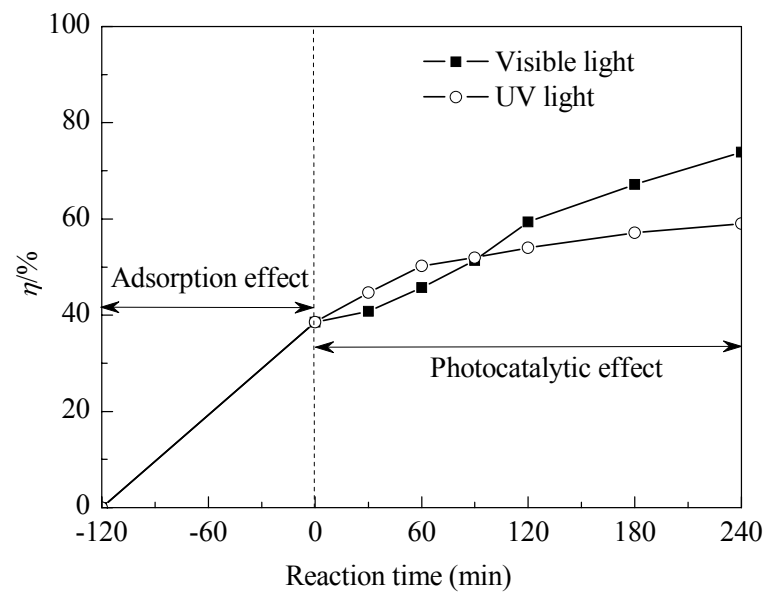

Fig. 9. Degradation efficiency of MB solution by the graphene-CdSe composite under irradiation of UV light and visible light. The concentration of organic dye was $50 \mu \mathrm{mol} / \mathrm{L}$. The amount of graphene-CdSe composite was $0.03 \mathrm{~g}$. 
pseudo-first order kinetics with respect to the concentration of MB:

$$
-\mathrm{d} c / \mathrm{d} t=k_{\text {app }} c
$$

Integration of the equation (with $c=c_{\text {ads }}$ at $t=0$, with $c_{\text {ads }}$ being the initial concentration in the bulk solution after dark adsorption and $t$ the reaction time) lead to

$$
-\ln \left(c / c_{\text {ads }}\right)=k_{\text {app }} t
$$

where $c$ and $c_{\text {ads }}$ are the reactant concentration at time $t=t$ and $t=0$, respectively, $k_{\text {app }}$ and $t$ are the apparent reaction rate constant and time, respectively. Accordingly, a plot of $-\ln \left(c / c_{\text {ads }}\right)$ versus $t$ will yield a slope of $k_{\text {app. }}$. The results are displayed in Fig. 10. The linearity of the plot suggested that the photodegradation reaction approximately followed pseudo-first order kinetics with $k_{a p p}$ of $0.002 \mathrm{~min}^{-1}$ and $0.0034 \mathrm{~min}^{-1}$ for degradation under UV light and visible light, respectively.

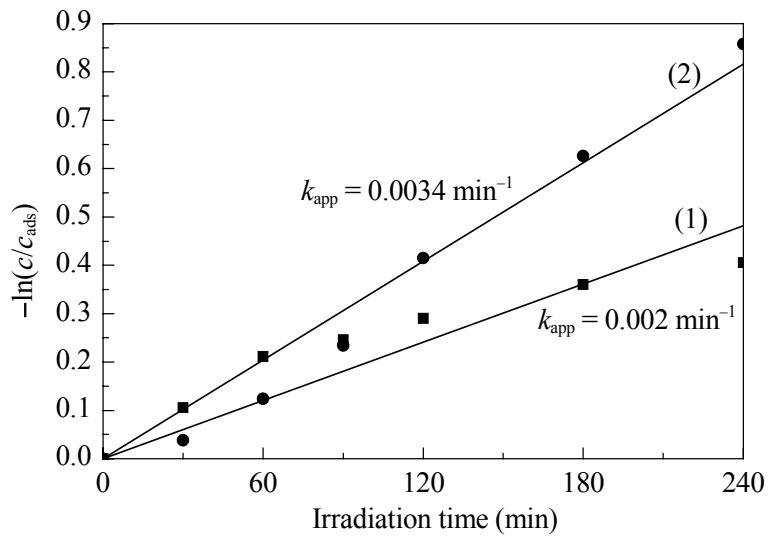

Fig. 10. Apparent first order kinetics, $-\ln \left(c / c_{a d s}\right)$ vs $t$, for MB degradation on graphene-CdSe composite under UV light (1) and visible light (2) irradiation. The concentration of MB solution was $50 \mu \mathrm{mol} / \mathrm{L}$. The amount of catalyst was $0.03 \mathrm{~g}$.

The scheme for the excitation and charge transfer process between CdSe particles and graphene nanosheets under light irradiation is shown in Fig. 11. Under irradiation by visible lamp, the graphene nanosheets act as good electron acceptors [15,36-38] and accept the electrons produced by light irradiation. Meanwhile, the $\mathrm{CdSe}$ can be also excited to produce electrons and holes in the conduction band (CB) and valence band (VB) of CdSe. Then the electrons accepted by the graphene nanosheets from light excitation can transfer into the $\mathrm{CB}$ of $\mathrm{CdSe}$, thereby increasing the number of electrons as well as the rate of electron-induced redox reactions. The generated electrons $\left(\mathrm{e}^{-}\right)$react with dissolved oxygen molecules and produce oxygen peroxide radicals $\mathrm{O}_{2}{ }^{--}$. The positive charged hole $\left(\mathrm{h}^{+}\right)$can react with the $\mathrm{OH}^{-}$ derived from $\mathrm{H}_{2} \mathrm{O}$ to form hydroxyl radicals $\mathrm{OH}^{*}$. The $\mathrm{MB}$ molecules then can be photocatalytically degraded by the oxygen peroxide radicals $\mathrm{O}_{2}{ }^{-}$and hydroxyl radicals $\mathrm{OH}^{*}$ to $\mathrm{CO}_{2}, \mathrm{H}_{2} \mathrm{O}$, and other mineralization products.

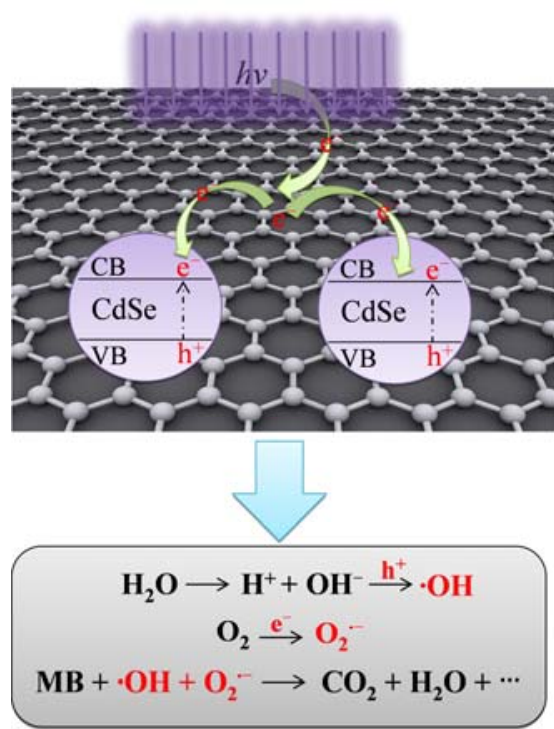

Fig. 11. Graphene acting as a 2D conducting support for CdSe particles.

\section{Conclusions}

A graphene-CdSe composite was successfully synthesized by a simple hydrothermal method. Only the cubic crystal structure of CdSe was present on the graphene-CdSe composite. The surface of the graphene was coated with CdSe particles. C, Cd, and Se present with high contents in the composite. The graphene-CdSe composite had strong photoabsorption in the UV light and visible light range. The photocatalytic activity of the graphene-CdSe composite was investigated by the degradation of $\mathrm{MB}, \mathrm{MO}$, and $\mathrm{Rh} . \mathrm{B}$ in aqueous solution under UV or visible light irradiation. The graphene-CdSe composite exhibited excellent photocatalytic activity for the degradation of MB solution under visible light irradiation.

\section{Acknowledgements}

This work was supported by Research Foundation of Hanseo University in 2011. The authors are grateful to the University for financial support.

\section{References}

1 Robel I, Bunker B A, Kamat P V. Adv Mater, 2005, 17: 2458

2 Yu J G, Ma T T, Liu G, Cheng B. Dalton Trans, 2011, 25: 6635

3 Yu J G, Ma T T, Liu S W. Phys Chem Chem Phys, 2011, 13: 3491

4 Oh W C, Zhang F J, Chen M L. J Ind Eng Chem, 2010, 16: 299

5 Zhu L, Meng Z D, Oh W C. Chin J Catal, 2011, 32: 746

6 Novoselov K S, Geim A K, Morozov S V, Jiang D, Zhang Y, 
Dubonos S V, Grigorieva I V, Firsov A A. Science, 2004, 306: 666

7 Geim A K, Novoselov K S. Nat Mater, 2007, 6: 183

8 Meyer J C, Geim A K, Katsnelson M I, Novoselov K S, Booth T J, Roth S. Nature, 2007, 446: 60

9 Zhu Y, Murali S, Cai W, Li X, Suk J W, Potts J R, Ruoff R S. Adv Mater, 2010, 22: 3906

10 Wang X, Li X, Zhang L, Yoon Y, Weber P K, Wang H, Guo J, Dai H. Science, 2009, 324: 768

11 Xia J, Chen F, Li J, Tao N. Nat Nanotechnol, 2009, 4: 505

12 Chen F, Qing Q, Xia J, Li J H, Tao N. J Am Chem Soc, 2009, 131: 9908

13 Tang L, Wang Y, Li Y, Feng H, Lu J, Li J H. Adv Funct Mater, 2009, 19: 2782

14 Li Y, Tang L, Li J H. Electrochem Commun, 2009, 11: 846

15 Williams G, Seger B, Kamat P V. ACS Nano, 2008, 2: 1487

16 Xiong Z G, Zhang L L, Ma J Z, Zhao X S. Chem Commun, 2010, 46: 6099

17 Zhang J T, Xiong Z G, Zhao X S. J Mater Chem, 2011, 21: 3634

18 Kamat P V. Chem Rev, 1993, 93: 267

19 Su B, Choy K L. Thin Solid Films, 2000, 361-362: 102

20 Murali K R, Swaminathan V, Trivedi D C. Sol Energy Mater Sol Cells, 2004, 81: 113

21 Patil K R, Paranjape D V, Sathaye S D, Mitra A, Padalkar S R, Mandale A B. Mater Lett, 2000, 46: 81

22 Ahmadi T S, Wang Z L, Green T C, Henglein A, El-Sayed M A. Science, 1996, 272: 1924

23 Huynh W U, Peng X, Alivisatos A P. Adv Mater, 1999, 11: 923
24 Elmalem E, Saunders A E, Costi R, Salant A, Banin U. $A d v$ Mater, 2008, 20: 4312

25 Costi R, Saunders A E, Elmalem E, Salant A, Banin U. Nano Lett, 2008, 8: 637

26 Shen X C, Zhang Z L, Zhou B, Peng J, Xie M, Zhang M, Pang D W. Environ Sci Technol, 2008, 42: 5049

27 Oh W C, Chen M L, Zhang K, Zhang F J, Jang W K. J Korean Phys Soc, 2010, 56: 1097

28 Oh W C, Zhang F J, Chen M L. J Ind Eng Chem, 2010, 16: 321

29 Wang T T, Wang J L, Zhu Y C, Xue F, Cao J, Qian Y T. J Phys Chem Solids, 2010, 71: 940

30 Raevskaya A E, Stroyuk A L, Kuchmiy S Ya, Azhniuk Yu M, Dzhagan V M, Yukhymchuk V O, Valakh M Ya. Colloid Surf A, 2006, 290: 304

31 Li Q, Guo B D, Yu J G, Ran J R, Zhang B H, Yan H J, Gong J R. J Am Chem Soc, 2011, DOI: 10.1021/ja2025454

32 Xiang Q J, Yu J G, Jaroniec M. J Phys Chem C, 2011, 115: 7355

33 Chen M L, Zhang F J, Zhang K, Meng Z D, Oh W C. J Photocatal Sci, 2010, 1: 19

34 Oh W C. J Photocatal Sci, 2010, 1: 29

35 Li Y, Li X, Li J, Yin J. Water Research, 2006, 40: 1119

36 Novoselov K S, Geim A K, Morozov S V, Jiang D, Katsnelson M I, Grigorieva I V, Dubonos S V, Firsov A A. Nature, 2005, 438: 197

37 Williams G, Kama P V. Langmuir, 2009, 25: 13869

38 Stankovich S, Dikin D A, Dommett G H B, Kohlhass K M, Zimney E J, Stach E A, Piner R D, Nguyen S T, Ruoff R S. Nature, 2006, 442: 282 